\title{
Verbundausbildung am Zentrum für Mikroproduktion e.V.
}

\author{
R. Labes ${ }^{1}$, M. Leester-Schädel ${ }^{2}$ \\ ${ }^{1}$ eck*cellent IT GmbH, \\ Theodor-Heuss-Straße 2, 38122 Braunschweig \\ Deutschland \\ ${ }^{2}$ Zentrum für Mikroproduktion e.V., \\ c/o Institut für Mikrotechnik \\ Technische Universität Braunschweig \\ Alte Salzdahlumer Str. 203, 38124 Braunschweig \\ Deutschland
}

\section{$1 \quad$ Einleitung}

Der Beruf der Mikrotechnologin/ des Mikrotechnologen wird in den Bereich der Hochtechnologieberufe eingegliedert. Damit soll zum Ausdruck kommen, dass sich die Tätigkeiten der Mikrotechnologen und damit das ganze Berufsbild entsprechend der Technologieentwicklungen seit der Entstehung des Berufs im Jahr 1998 rasant geändert haben und diese Entwicklung voraussichtlich noch weit in die Zukunft hinein reichen wird. Das bedeutet auch, dass die Inhalte der gewerblichen Ausbildung stets aktualisiert und immer neue Fachrichtungen integriert werden müssen. Gleichzeitig sind viele Mikrotechnik-Firmen außerordentlich spezialisiert. Dies hat auf der einen Seite zur Folge, dass die Anforderungen an die Fachkräfte sehr hoch und vielfältig sind. Auf der anderen Seite sehen sich diese Firmen häufig nicht in der Lage selbst auszubilden, da ihnen ein großer Teil der Ausstattung zur Vermittlung der geforderten betrieblichen Lehrinhalte nicht zur Verfügung steht.

In Braunschweig wurde daher fast von Beginn der Ausbildung von Mikrotechnologen an eine intensive Zusammenarbeit der ausbildenden Einrichtungen realisiert. Ziel ist dabei, hochqualifizierte und hochflexible Fachkräfte auszubilden, Ausbildungs- und Arbeitsplätze zu schaffen und Betriebe und Forschungseinrichtungen bei der Ausbildung bedarfsgerecht zu unterstützen. Im folgenden Text werden die Entwicklung der Verbundausbildung im Zentrum für Mikroproduktion e.V., ihre inhaltliche und zeitliche Konzeptionierung sowie erste Erfahrungen und zukünftige Ziele beschrieben.

\section{Hintergrund}

\subsection{Das Zentrum für Mikroproduktion e.V.}
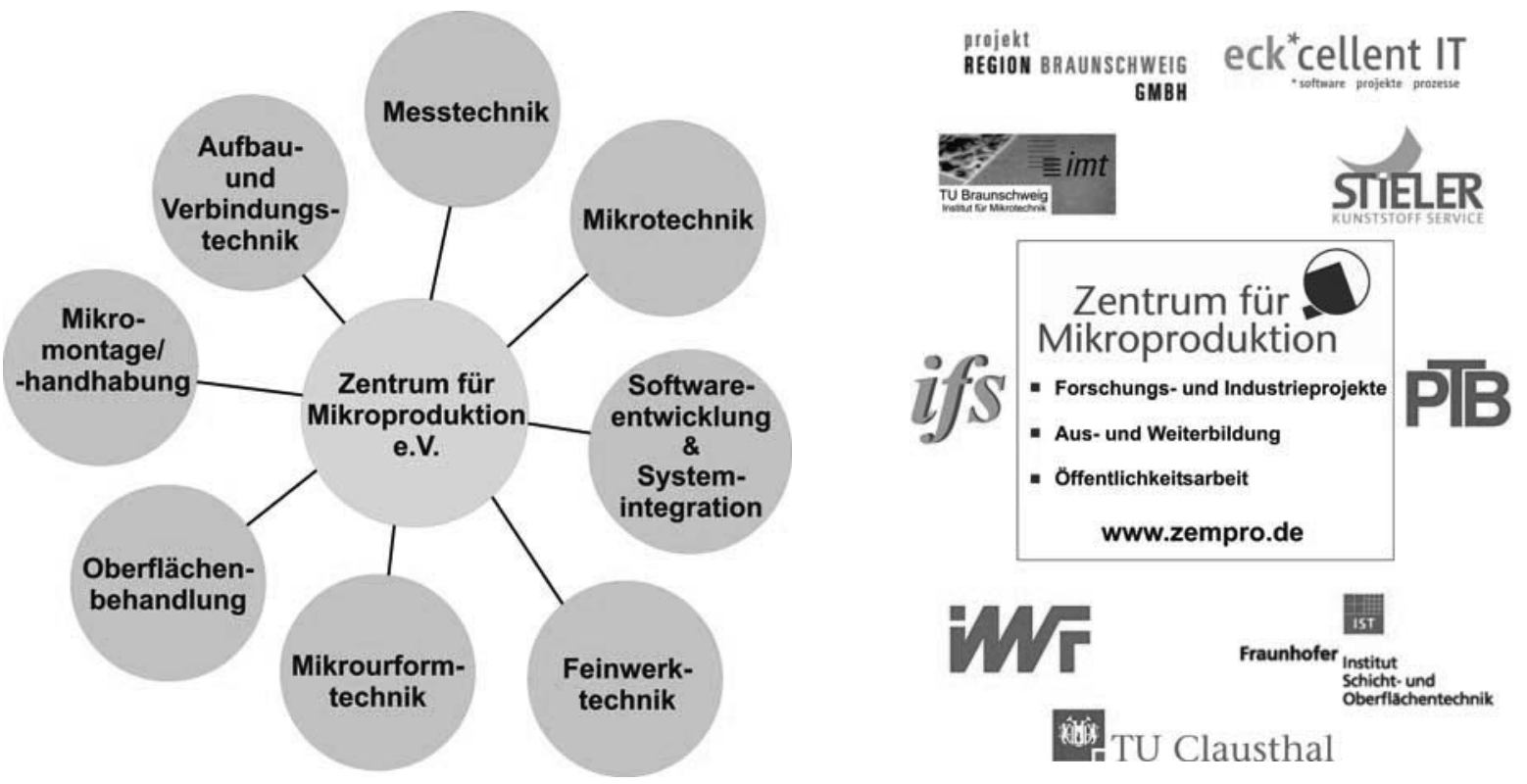

Bild 1 links: Kompetenzen im Zentrum für Mikroproduktion, rechts: Derzeitige Mitgliedsinstitutionen 
Das Zentrum für Mikroproduktion (ZeMPro) wurde im Jahr 2005 als Verein gegründet und versteht sich als Kompetenzzentrum im Bereich der Mikroproduktion. Die Kompetenzen umfassen Forschung und Entwicklung sowie den Transfer in die industrielle Anwendung (Bild 1, links). Ziel ist es, regionale Wertschöpfungsketten aufzubauen und diese innerhalb des Zukunftsmarktes Mikrosystemtechnik nachhaltig zu positionieren.

Partner des Zentrums sind regionale Unternehmen sowie Institute der Technischen Universität Braunschweig, der Technischen Universität Clausthal, der Fraunhofer-Gesellschaft sowie der Physikalisch-Technischen Bundesanstalt (Bild 1, rechts).

\section{2 eck*cellent IT GmbH}

eck*cellent IT ist ein international tätiger, expandierender Anbieter von Individual-Softwareentwicklung. Die Kombination aus Fach- und Sozialkompetenz, umfangreichen Erfahrungen im Projektmanagement auch von Großprojekten- und stets transparenter Kommunikation zeichnen das Unternehmen aus. Weitere Schwerpunkte von eck*cellent IT sind die Analyse von Geschäftsprozessen, die Dokumentation von Anforderungen und die Entwicklung von Methoden, um Abläufe zu optimieren.

Durch das Engagement in Forschungsprojekten und durch die intensive Verankerung in innovativen Bereichen ist eck*cellent IT früher als andere in neue Trends und Entwicklungen eingebunden. Es ist das Ziel, als Partner im Zentrum für Mikroproduktion e.V. an neuen Technologien mitzuwirken und innovative Ideen zu unterstützen. eck* cellent IT ist in diesem Verbund der Partner für die Softwareentwicklung und Systemintegration. Nach der reinen „Produktion“ der Mikrosysteme sind Funktionen notwendig, um Daten - zum Beispiel aus Sensoren - zu generieren, zu übertragen und zu speichern. Erfassung, Monitoring und Darstellung der Daten sind dabei die Schwerpunke der eck* ${ }^{*}$ ellent IT GmbH.

Dieses spezielle Wissen soll in die Verbundausbildung eingebracht und weiter vermittelt werden.

\subsection{Ausbildung zur Mikrotechnologin/ zum Mikrotechnologen in Braunschweig}

Das Institut für Mikrotechnik (IMT) der TU Braunschweig begann 1999 mit der Ausbildung von Mikrotechnologen und stellt inzwischen jedes Jahr einen Ausbildungsplatz zur Verfügung. Da eine Übernahme der Auszubildenden häufig nicht möglich ist, wird verstärkt auf breitbandige Ausbildungsinhalte Wert gelegt, um dem gesellschaftlich bestehenden Bedarf gerecht zu werden. Zu Beginn der Ausbildungsaktivität am IMT war ein hohes Maß an Eigeninitiative gefordert. Da der Ausbildungsberuf erst ein Jahr zuvor eingerichtet wurde, bestanden kaum Erfahrungen und Orientierungshilfen für die Gestaltung des betrieblichen Teils der Ausbildung. Das IMT hat daher zunächst alleine und ab dem zweiten Jahrgang gemeinsam mit der Physikalisch Technischen Bundesanstalt PTB den innerbetrieblichen Lehrplan erstellt. Der Verbund mit der PTB machte es schnell und unbürokratisch möglich, einige Ausbildungsinhalte aufzuteilen und die Auszubildenden beider Einrichtungen zeitweilig gemeinsam zu schulen. Beide Einrichtungen haben dann zusammen mit der IHK Braunschweig die Prüfungskommission für die Bewertung der Abschlussprüfung gebildet, um die jeweiligen betrieblichen Ausbildungsinhalte, die unmittelbar mit der technologischen Ausstattung der Einrichtung verknüpft sind, zu berücksichtigen.

In den darauffolgenden Jahren entschieden sich weitere Forschungseinrichtungen und Firmen in der Region für die Ausbildung von Mikrotechnologen, die zusammen mit dem IMT und der PTB im Ausbildungsnetzwerk mst niedersachsen kooperierten (Bild 2). Die Erfahrungen während der Laufzeit des Netzwerkes waren außerordentlich positiv und es hat sich gezeigt, dass die Kombination von Forschungseinrichtungen und Industriebetrieben innerhalb eines Ausbildungsverbundes von großem Vorteil sowohl für die Auszubildenden als auch für die Ausbilder ist: Die Auszubildenden haben die Möglichkeit, sich weit über die Grenzen des eigenen Ausbildungsbetriebes hinaus zu informieren, andere Betriebsformen und Tätigkeiten kennenzulernen und Fachkenntnisse zu erwerben. Die Betriebe erhalten durch den Verbund Partner, mit denen eine eigene Ausbildung erst möglich wird und können dadurch hochqualifizierte Nachwuchskräfte gewinnen. 

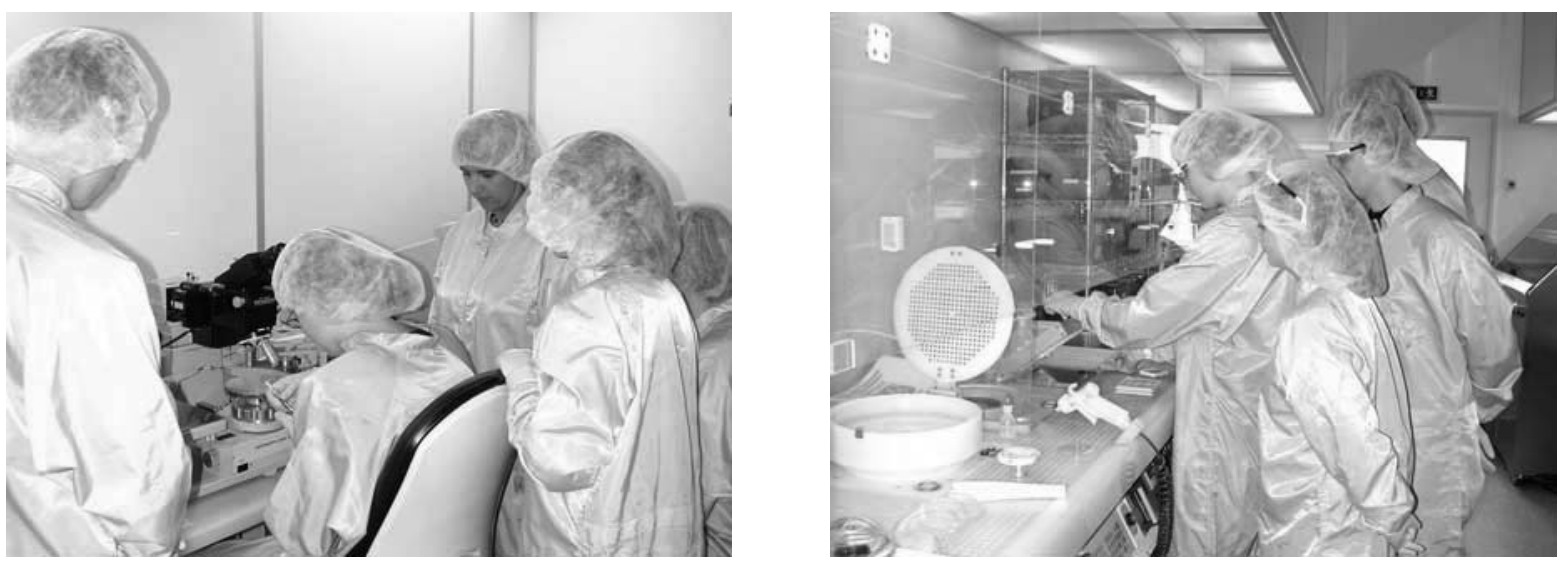

Bild 2 Lehrgang am IMT im Rahmen der Kooperation im Ausbildungsnetzwerk mst niedersachsen

\section{Verbundausbildung im Zentrum für Mikroproduktion e.V.}

\subsection{Ausgangssituation}

Auf Initiative des Zentrums für Mikroproduktion e.V. und unter Federführung des IMT (Gestaltung der Ausbildungsinhalte, zeitliche und fachliche Zuordnung zu den beteiligten Partnern), formierte sich für den Ausbildungsjahrgang 2007 ein neuer Verbund. Integriert sind insgesamt acht Vereinsmitglieder aus Forschung und Industrie, die mit Ausnahme des IMT im Bereich der Mikrosystemtechnik selbst noch nicht ausgebildet haben.

Die Mitglieder des Zentrums für Mikroproduktion e.V. sind über den fachlichen Kontext in der Region miteinander verknüpft, so dass das Engagement zur Ausbildung ein kleiner Schritt war, zumal zunächst nur das IMT eine Auszubildende eingestellt hat. Die übrigen Institute und Betriebe werden jeweils 2-3 Wochen in die praktische Ausbildung im ersten und zweiten Ausbildungsjahr mit sogenannten Ausbildungsmodulen eingebunden. Im dritten Ausbildungsjahr erfolgt die Vorbereitung für die Abschlussprüfung gezielt am IMT.

Die schulische Ausbildung findet an der Beruflichen Schule des Kreises Steinburg, Itzehoe, statt. Dies ist möglich, da es in Niedersachsen für so genannte Splitter- oder Minderheitenberufe die Möglichkeit zur Wahl der Berufsschule, auch Bundesländer übergreifend, gibt. Durch die sehr enge Zusammenarbeit und die regelmäßige, gegenseitige Abstimmung der Lehrinhalte wird der Ausbildungsverbund von der Berufsschule sehr unterstützt. Der Berufschulunterricht findet in mehrwöchigen Blöcken statt, so dass auch die zeitliche Organisation der Ausbildungsmodule erst möglich wird.

\subsection{Motivation und Ziele}

Die Motivation für den neuen Verbund war zum einen der gesellschaftliche Bedarf zur Ausbildung junger Menschen. Zum anderen können innovative Technologien über den Verbund in einem wesentlich höheren Maße als in einem Einzelbetrieb in die Ausbildung integriert werden. Dies wird besonders deutlich, wenn man die jeweiligen Kompetenzen der einzelnen Mitglieder des Zentrums für Mikroproduktion e.V. betrachtet. Diese reichen von der Silizium-Bulkmikromechanik, der Halbleitertechnologie, dem Mikrofügen und dem Mikrospritzguss über die Mikrofluidik, die Mikrooptik und die Oberflächtentechnik bis hin zur Messtechnik sowie zur Softwareentwicklung und Systemintegration. Die Auszubildenden erhalten durch den Verbund vielfältige und anspruchsvolle Fachkenntnisse aus mehreren, innovativen Spezialbereichen, die sie für ihre spätere berufliche Tätigkeit in einem besonderen Maße qualifizieren. Dies ist besonders dann sinnvoll, wenn die Ausbildung nicht für den Eigenbedarf geschieht. Durch die modulare Form der Verbundausbildung können Ausbildungsinhalte kurzfristig und stetig aktualisiert werden, und sie können darüber hinaus auf die speziellen Fähigkeiten und Interessen der einzelnen Auszubildenden angepasst werden.

Ziel des Ausbildungsverbundes ist darüber hinaus, den Beruf der Mikrotechnologin/des Mikrotechnologen bei den Verbundpartnern bekannt zu machen und damit langfristig und nachhaltig neue Arbeits- und Ausbildungsplätze zu schaffen. Die Verbundausbildung dient auch dazu, die Vereinsmitglieder aber auch andere Unternehmen und Forschungseinrichtungen der Region zu unterstützen, indem innen der Zugriff auf hochqualifizierte Fachkräfte ermöglicht wird, oder indem sie selbst schrittweise an die Ausbildung eigener Fachkräfte herangeführt werden. 
Ein weiterer positiver Effekt der Verbundausbildung ist der Transfer von Wissen und Erfahrungen sowie die Bündelung von Kompetenzen. Über die gewerbliche Ausbildung eröffnet sich ein weiterer Weg, um auf wissenschaftlicher und betrieblicher Ebene die bestehenden Kontakte zu vertiefen und voneinander zu profitieren. Auf der anderen Seite ist natürlich auch zu berücksichtigen, dass das notwendige Maß an Vertraulichkeit eingehalten wird.

Nicht zuletzt werden durch die genannten Möglichkeiten und Ziele herausragende Standortfaktoren geschaffen. Die Verbundausbildung stärkt das Netzwerk an sich und bietet eine Basis zur Erweiterung, auch zum Beispiel durch die gemeinsame Öffentlichkeitsarbeit.

\subsection{Aufbau der Verbundausbildung und Konzept}

Da das IMT als bisher einzige Einrichtung des Zentrums für Mikroproduktion e.V. Mikrotechnologen ausbildete, haben die dortigen Ausbildungsverantwortlichen die Organisation und Gestaltung der Verbundausbildung übernommen.

Der Aufbau der Verbundausbildung begann mit ausführlichen Gesprächen mit den einzelnen Ausbildungspartnern. Darin wurden die Module, deren Umfang und Inhalt sowie eine vorläufige zeitliche Reihenfolge diskutiert. Häufig haben die Ausbildungspartner dabei auf bestehende Lehrgänge zurückgegriffen, die sie beispielsweise für Studierende oder Auszubildende anderer Fachrichtungen anbieten, und haben diese bei Bedarf an die Ausbildung von Mikrotechnologen angepasst.

Nachdem sich schnell herauskristallisierte, dass der Verein großes Interesse an der Schaffung eines Verbundausbildungsplatzes hat und sich auch nahezu alle Vereinsmitglieder mit einem oder mehreren Modulen daran beteiligen werden, wurde eine Anfrage an die TU Braunschweig hinsichtlich der Verfügbarkeit eines Ausbildungsplatzes gerichtet. Auch hier stieß die Aktivität auf positive Resonanz, so dass die Finanzierung schnell geklärt werden konnte.

Die weiterführende Organisation umfasste auch die Klärung verschiedener Modalitäten, wie z.B. die Unterzeichnung von Geheimhaltungserklärungen, die Sicherstellung von geeigneten Unterkünften für die Auszubildende/den Auszubildenden, während sie in den Firmen und Forschungseinrichtungen außerhalb Braunschweigs sind, die Finanzierung von Fahrtkosten etc.. Kurz vor den jeweiligen Modulen findet noch einmal ein Treffen mit den Beteiligten statt, um die konkrete Feinplanung abzuschließen.

Folgende Module wurden bisher in die Verbundausbildung integriert (Tabelle 1): 


\begin{tabular}{|c|c|c|c|}
\hline Modul & Inhalt & Dauer & Zeitplan \\
\hline Reinraumtechnik & $\begin{array}{l}\text { - Aufbau eines Reinraums, Reinraum-Konzepte } \\
\text { - Peripherie und Produktionsanlagen } \\
\text { - Serhalten im Reinraum, Sicherheitsmaßnahmen und Überprüfen der Reinraum-Bedingungen } \\
\text { - Wartung von Anlagen }\end{array}$ & $\begin{array}{c}2-3 \\
\text { Wochen }\end{array}$ & \multirow{6}{*}{$\begin{array}{l}1 . \\
\text { Ausb.- } \\
\text { jahr }\end{array}$} \\
\hline $\begin{array}{l}\text { Werkstoffe und } \\
\text { Analytik }\end{array}$ & $\begin{array}{l}\text { - Werkstoffe und ihre Eigenschaften } \\
\text { - Bearbeitungsmöglichkeiten } \\
\text { - Prüfverfahren zur metallkundlichen Charakterisierung } \\
\text { - Einsatzbereiche }\end{array}$ & $\begin{array}{c}3 \\
\text { Wochen }\end{array}$ & \\
\hline CAD & $\begin{array}{l}\text { - Grundlagen des technischen Zeichnens } \\
\text { - Einarbeitung in Solid Works und Corel Draw } \\
\text { - Darstellung eines konkreten Bauteils }\end{array}$ & 1 Woche & \\
\hline $\begin{array}{l}\text { Zerspanende } \\
\text { Metall- } \\
\text { bearbeitung }\end{array}$ & $\begin{array}{l}\text { - Grundlagen spanende Metallbearbeitung } \\
\text { - Fertigung eines Drehteils }\end{array}$ & 1 Woche & \\
\hline $\begin{array}{l}\text { Zerspanende } \\
\text { Mikrobearbeitung }\end{array}$ & $\begin{array}{l}\text { - Mikrozerspanung } \\
\text { - Qualitätsüberwachung }\end{array}$ & 1 Woche & \\
\hline $\begin{array}{l}\text { Mikro- } \\
\text { Handhabungs- } \\
\text { technik }\end{array}$ & $\begin{array}{l}\text { - Handhabungstechnik } \\
\text { - Mikromontage }\end{array}$ & 1 Woche & \\
\hline E-Technik & - Grundlagen der Elektronik, Theorie und Praxis & $\begin{array}{c}4 \\
\text { Wochen }\end{array}$ & \multirow{4}{*}{$\begin{array}{l}2 . \\
\text { Ausb.- } \\
\text { jahr }\end{array}$} \\
\hline $\begin{array}{l}\text { Dünnschicht- } \\
\text { technik, } \\
\text { Plasmatechnik }\end{array}$ & $\begin{array}{l}\text { Diamanttechnologie, Siliziumbeschichtung: } \\
\text { Aufdampfen, PECVD, HFCVD, Sputtern, spez. } \\
\text { Hochpulssputtern } \\
\text { - } \text { Messverfahren } \\
\text { - Plasmatechnik } \\
\text { - Superharte Schichten: Schichtarten, Eigenschaften, } \\
\text { Einsatzbereiche, Prüfverfahren } \\
\text { - Umsetzung der Theorie am Bsp. Diamantschichten }\end{array}$ & $\begin{array}{c}6 \\
\text { Wochen }\end{array}$ & \\
\hline $\begin{array}{l}\text { Aufbau- und } \\
\text { Verbindungs- } \\
\text { technik, } \\
\text { Mikrofügen }\end{array}$ & $\begin{array}{l}\text { - Grundlagen zu Aufbau- und Verbindungstechniken } \\
\text { - } \text { Klebstoffe } \\
\text { - } \text { Mikrokleben } \\
\text { - Lastragstechniken, Mikrodosierverfahren } \\
\text { - } \text { Mikrolaserstrahlschweißen } \\
\end{array}$ & $\begin{array}{c}3 \\
\text { Wochen }\end{array}$ & \\
\hline $\begin{array}{l}\text { Qualitäts- } \\
\text { management }\end{array}$ & $\begin{array}{l}\text { - Zertifizierung als Fundament des } \\
\text { Qualitätsmanagements } \\
\text { - Zertifizierungsverfahren } \\
\text { - } \text { Definition von Prozessen } \\
\text { - Programmiersprachen am Bsp. C } \\
\text { - Anwendung von relevanter Standardsoftware } \\
\end{array}$ & $\begin{array}{c}3 \\
\text { Wochen }\end{array}$ & \\
\hline $\begin{array}{l}\text { Extrusion, } \\
\text { Spritzgießen und } \\
\text { Prüfen }\end{array}$ & $\begin{array}{l}\text { - Kunststoffe (Thermoplaste, Duroplaste) } \\
\text { - Herstellen, Aufbereiten } \\
\text { - Extrudieren } \\
\text { - Pritzgießen } \\
\text { - } \text { Beispiel: Vom Rohstoff zum Spritzgussbauteil } \\
\end{array}$ & $\begin{array}{c}3 \\
\text { Wochen }\end{array}$ & $\begin{array}{l}\text { Zu } \\
\text { Beginn } \\
\text { des } 3 . \\
\text { Ausb.- } \\
\text { jahres }\end{array}$ \\
\hline
\end{tabular}

Tabelle 1: Module der Verbundausbildung am Zentrum für Mikroproduktion e.V.

Die eck*cellent IT GmbH übernimmt das Modul Qualitätsmanagement. Zusätzlich findet eine Einweisung in Programmiersprachen und Standardprogramme statt.

Für Mikrotechnologen ist es wichtig, Tätigkeiten zur Qualitätssicherung durchzuführen und ihre Arbeit sorgfältig zu dokumentieren. Dazu gibt eck*cellent IT in den drei Wochen die nötigen Werkzeuge wie Textverarbeitungs- und Tabellenkalkulationsprogramme an die Hand und ermöglicht den 
Auszubildenden, grundlegende Prozessabläufe kennen zu lernen und selbstständig formulieren zu können.

Für die Tätigkeiten im Bereich Mikroproduktion, die oft einen großen Forschungsanteil haben, ist der Einsatz von neuesten Technologien und Kenntnissen in der Erstellung von Hilfsprogrammen häufig notwendig. Im Rahmen der Ausbildung soll deshalb eine Einweisung in dafür sinnvolle Programmiersprachen vermittelt werden.

Durch den Einblick des Auszubildenden / der Auszubildenden in ein privatwirtschaftliches Unternehmen wird die Möglichkeit der Zusammenarbeit der Institute und Unternehmen gefördert. Ein Mikrotechnologe der Verbundausbildung lernt Arbeitsweisen und Abläufe sowohl in der Privatwirtschaft - speziell am Beispiel eines Dienstleisters im IT-Bereich- als auch in Forschungseinrichtungen kennen. Durch diese Kombination wird bei den Auszubildenden ein übergreifendes Verständnis für die Bereiche erreicht, um zukünftig gemeinsame Projekte erkennen und umsetzen zu können.

Zur Evaluierung der vermittelten Inhalte fertigen die Auszubildenden nach jedem Modul eine schriftliche Zusammenfassung über ihre Tätigkeiten an und halten zusätzlich einen entsprechenden, etwa halbstündigen Vortrag. Der Vortrag dient dabei einerseits zur Vorbereitung der Auszubildenden auf ihre mündliche Abschlussprüfung und andererseits für den Austausch von Erfahrungen und Erkenntnissen unter den Partnern der Verbundausbildung. Die Qualität der Module wird außerdem über regelmäßige Absprachen der Verantwortlichen überprüft und ggf. optimiert. Am Ende ihrer Ausbildung erhalten die Auszubildenden ein Zertifikat über alle geleisteten Module und können dies ihren Bewerbungen beifügen.

\subsection{Bisherige Erfahrungen, zukünftige Ziele}

Der aktuell laufende, erste Verbundausbildungsjahrgang hat bisher ausnahmslos positive Erfahrungen ergeben, und zwar sowohl bezüglich der organisatorischen wie auch der inhaltlichen Punkte. Die Vorträge der Auszubildenden zeigten, wie umfangreich das vermittelte Wissen trotz der im Verhältnis kurzen Module sein kann und mit wie viel Interesse alle Beteiligten dazu beigetragen haben. Alle Ausbildungspartner haben außerdem unbürokratische Lösungen z.B. hinsichtlich der Arbeitszeit der Auszubildenden gefunden, um die ungewohnten Anfahrtswege etc. zu berücksichtigen.

Allerdings stellt dieses Ausbildungsmodell auch besondere Anforderungen an die Auszubildenden: Eine überdurchschnittlich hohe Flexibilität sowie die Fähigkeit auf Menschen zugehen zu können hat sich als sehr wichtig bestätigt. Die Auszubildenden müssen sich schnell und kurzfristig auf neue Situationen, fremde Vorgesetzte und Kollegen einstellen. Diese Anforderungen bieten aber auch die Chance für die Auszubildenden, sich schneller und intensiver hinsichtlich ihrer sozialen Kompetenzen weiterzuentwickeln.

Dass die Verbundausbildung die Kommunikation der daran beteiligten Partner stärken wird, haben bereits Erfahrungen mit einer ehemaligen Auszubildenden gezeigt. Sie wechselte nach ihrer Ausbildung vom IMT in das Fraunhofer Institut für Schicht- und Oberflächentechnik und nutzt nach wie vor verschiedene Technologien des IMT und umgekehrt. Die Zusammenarbeit ist sehr effizient und unkompliziert.

Das unmittelbare Ziel ist, die Verbundausbildung für den Jahrgang 2010 erneut durchzuführen. Abhängig von der endgültigen Evaluation wird dies mit derselben inhaltlichen und zeitlichen Abfolge geschehen. Die Ausbildungspartner haben bereits ihre erneute Teilnahme signalisiert. Darüber hinaus wird angestrebt, eine erweiterte Verbundausbildung zu initiieren. Damit ist gemeint, dass bisher passive Ausbildungspartner selbst einen Ausbildungsplatz zur Verfügung stellen und diesen in die Verbundausbildung integrieren. Damit würden weitere Ausbildungsplätze geschaffen.

Das IMT wird bei der Einrichtung neuer Ausbildungsplätze unterstützen und beraten. Die Suche nach geeigneten Bewerberinnen und Bewerbern sowie die anschließende Auswahl der Bewerber können auf Wunsch gemeinsam erfolgen.

\section{$4 \quad$ Zusammenfassung}

In diesem Beitrag wurde die Verbundausbildung von Mikrotechnologen vorgestellt, an der die Mitglieder des Zentrums für Mikroproduktion e.V. beteiligt sind. Dazu wurden die Hintergründe, die Motivation und der organisatorische Aufbau des Ausbildungsmodells beschrieben.

In der bisherigen Laufzeit wurden die Erwartungen ausnahmslos erfüllt. Sowohl die beteiligten Ausbilder als auch die Auszubildende haben sehr positiv über die Module, die bereits stattgefunden haben, berichtet. Keiner der Ausbildungspartner hat sich bisher zurückgezogen, sodass einer Wiederholung bzw. einer Erweiterung der Verbundausbildung nichts entgegen steht. 